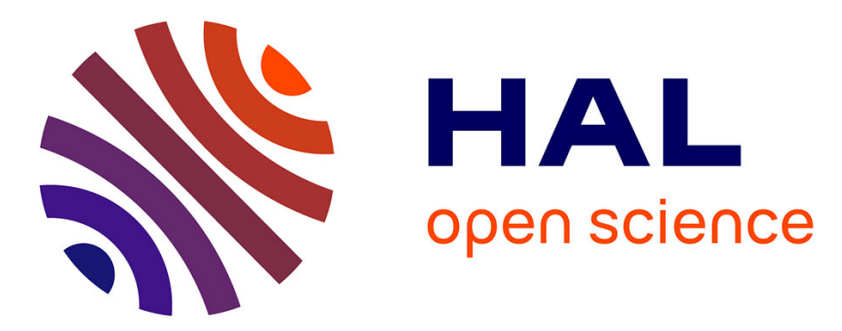

\title{
Low temperature attenuation of sound in restricted geometries
}

\author{
B. Lambert, D. Salin
}

\section{To cite this version:}

B. Lambert, D. Salin. Low temperature attenuation of sound in restricted geometries. Journal de Physique Lettres, 1980, 41 (20), pp.487-489. 10.1051/jphyslet:019800041020048700 . jpa-00231828

\section{HAL Id: jpa-00231828 https://hal.science/jpa-00231828}

Submitted on 1 Jan 1980

HAL is a multi-disciplinary open access archive for the deposit and dissemination of scientific research documents, whether they are published or not. The documents may come from teaching and research institutions in France or abroad, or from public or private research centers.
L'archive ouverte pluridisciplinaire HAL, est destinée au dépôt et à la diffusion de documents scientifiques de niveau recherche, publiés ou non, émanant des établissements d'enseignement et de recherche français ou étrangers, des laboratoires publics ou privés. 
Classification

Physics Abstracts

$62.60-67.20-67.70$

\title{
Low temperature attenuation of sound in restricted geometries
}

\author{
B. Lambert and D. Salin \\ Laboratoire d'Ultrasons ( ${ }^{*}$, Université Pierre-et-Marie-Curie, \\ Tour 13, 4 place Jussieu, 75230 Paris Cedex 05, France
}

(Reçu le $1^{\mathrm{er}}$ juillet 1980, accepté le 2 septembre 1980)

\begin{abstract}
Résumé. - Nous avons mesuré, à basses températures, l'atténuation du son à $1 \mathrm{GHz}$ pour de l'hélium liquide confiné dans les pores du verre de Vycor. Nos résultats montrent clairement le comportement à une dimension de la densité de masse normale dans cette géométrie réduite; la valeur mesurée est en accord avec les résultats d'expérience de $4^{\mathrm{e}}$ son; toutefois les valeurs expérimentales sont trois ordres de grandeur plus grandes que celles prédites théoriquement.
\end{abstract}

\begin{abstract}
We have measured the low temperature attenuation of sound at $1 \mathrm{GHz}$ in liquid helium confined in the pores of Vycor glass; our data clearly show the one-dimensional behaviour of the normal density in this restricted geometry; the experimental value is in agreement with fourth sound measurements; anyway the experimental values are three orders of magnitude larger than the theoretical one.
\end{abstract}

1. Introduction. - In the past few years there has been a great amount of work on the properties of systems depending on their dimensionality. For a system confined in a restricted geometry the size can become smaller than some characteristic length of the bulk and the properties of these systems are fairly modified. At low temperatures, in liquid helium, as the phonon thermal wavelength becomes larger than the pore radius or film thickness, the phase space of the phonons is reduced and thermodynamic quantities are affected.

In a previous paper [1] we have studied the critical attenuation of sound in liquid helium when the coherence length becomes of the order of magnitude of pore radius or film thickness. In this letter we report on low temperature measurements of the attenuation of sound in liquid helium confined in porous Vycor glass when the wavelength of typical thermal phonons becomes smaller than the pore radius. In a first step we recall some theoretical predictions; then we show how ultrasonic measurements is a test measurement of the normal density in restricted geometry; finally we present and discuss our experimental results.

2. Normal density in confined helium. - Basically the low temperature behaviour of the normal density

$\left(^{*}\right)$ Associated with the Centre National de la Recherche Scientifique. $\rho_{\mathrm{n}}$ in restricted geometry has been understood in the framework of the Landau concept [2] of the contribution of the elementary excitation spectrum to $\rho_{\mathrm{n}}$; at low temperatures $(T<1 \mathrm{~K})$ the only present elementary excitations are the phonons.

The walls in a restricted geometry reduce the phase space; in the simple case when the size $(R)$ (pore radius or film thickness) of the system is small compared to the wavelength $\lambda_{\mathrm{T}}$ of typical thermal phonons $\left(\pi \lambda_{\mathrm{T}} \gg R\right.$ with $\lambda_{\mathrm{T}}=\hbar_{\mathrm{c}} / k_{\mathrm{B}} T, c$ : velocity of sound) the temperature dependence of the contribution $\rho_{\mathrm{np}}^{[N]}$ of the phonons to the normal density $\rho_{\mathrm{n}}$ is [3] :

$$
\rho_{\mathrm{np}}^{[N]} \propto T^{N+1}
$$

where $N$ is the dimensionality of the restricted geometry (bulk $N=3$, film $N=2$, cylindrical pore $N=1$ ). Such a temperature dependence has been observed experimentally [4] but the experimental prefactor of eq. (1) is about three orders of magnitude larger than the predicted one $[3,5]$. The purpose of our experiment is then to test this prediction through ultrasonic measurements.

From elementary excitation interactions Khalatnikov et al. [6] have calculated the attenuation of sound in bulk liquid helium. At frequencies $\omega / 2 \pi \sim 1 \mathrm{GHz}$, the frequency $\omega / 2 \pi$ of the ultrasonic wave is much greater than the frequency $\tau^{-1}$ of the collisions between elementary excitations (phononphonon collision and phonon-roton collision). In this 
zero sound regime $(\omega \tau \gg 1)$ the attenuation of sound $\alpha_{\mathrm{He}}$ is independent of $\tau$ :

$$
\alpha_{\mathrm{He}}=\frac{3 \pi}{4}(u+1)^{2} \frac{\omega}{c} \frac{\rho_{\mathrm{np}}}{\rho}
$$

where $u$ is the Grüneisen $(u=2.84)$ constant and $\rho$ the density.

Due to the anomalous dispersion $[7,8]$ of saturated vapour pressure liquid helium, the processes which govern the attenuation of sound involve collisions of three phonons at small angle (for $T<0.6 \mathrm{~K}$ ); the equations which lead to eq. (2) are only dimensionality dependent through the normal density $\rho_{\text {np. }}$. So as far as the zero sound condition $(\omega \tau \gg 1)$ is fulfilled in the restricted geometry, expression (2) is still valid with the normal density $\rho_{\mathrm{np}}^{[N]}$ corresponding to the dimensionality $N$ of the restricted geometry :

$$
\alpha_{\mathrm{He}}^{[N]}=\frac{3 \pi}{4}(u+1)^{2} \frac{\omega}{c} \frac{\rho_{\mathrm{np}}^{[N]}}{\rho} .
$$

Tedious calculations [9] show that at $1 \mathrm{GHz}$ for a typical dimension of $100 \AA$ we are always in the zero sound regime. Thus ultrasonic measurement at high frequencies is a measurement of the normal density.

3. Experiment. - The restricted geometry we use is the tiny diameter pores of Vycor glass. The characterization of our samples have already been reported [1] we have two types of Vycor $\left(V_{1}\right.$ and $\left.V_{2}\right)$ with the same porosity $\left(x_{1}=x_{2}=x=31 \%\right)$ but with different pore diameters : $2 R_{1}=(130 \pm 10) \AA$ and $2 R_{2}=(75 \pm 5) \AA$. The ultrasonic measurement of the empty Vycor has been published in the preceding paper [1].

We have measured the attenuation of Vycor full of helium from $100 \mathrm{mK}$ to $1.5 \mathrm{~K}$ from $500 \mathrm{MHz}$ to $1100 \mathrm{MHz}$. As it was already noticed [1] it is not straightforward to deduce the attenuation of helium confined in Vycor from such a measurement : in a porous medium saturated with helium two dilatational waves can propagate [10], schematically one is at the velocity of the solid (Vycor), the other one is at the velocity of the fluid (helium).

These calculations have been recently reconsidered taking into account the attenuation of sound of the two media [11] : the authors used two propagation wave equations coupled by a friction $f$ proportional to the relative velocity of the two media (fluid $i=1$ and solid $\mathrm{i}=\mathrm{v})$ :

$$
\begin{aligned}
& \rho_{1} \ddot{r}_{1}=a_{1} \nabla^{2} \dot{r}_{1}+E_{1} \nabla^{2} r_{1}+f\left(\dot{r}_{\mathrm{v}}-\dot{r}_{1}\right) \\
& \rho_{\mathrm{v}} \ddot{r}_{\mathrm{v}}=a_{\mathrm{v}} \nabla^{2} \dot{r}_{\mathrm{v}}+E_{\mathrm{v}} \nabla^{2} r_{\mathrm{v}}+f\left(\dot{r}_{1}-\dot{r}_{\mathrm{v}}\right)
\end{aligned}
$$

where $r_{\mathrm{i}}$ is the displacement, $E_{\mathrm{i}}$ is the elastic modulus defined from the volocity $E_{\mathrm{i}}=\rho_{\mathrm{i}} v_{\mathrm{i}}^{2}$ and the attenuation of each medium alone has the form

$$
\alpha_{\mathrm{i}}=\frac{\omega^{2}}{2 \rho_{\mathrm{i}} v_{\mathrm{i}}^{3}} a_{\mathrm{i}}
$$

In the case we study the viscous penetration length of the sound wave $\left(\delta=\left(\frac{2 \eta}{\omega \rho_{\mathrm{n}}}\right)^{1 / 2}\right.$ with $\eta$, viscosity of helium, $\eta \gtrsim 1000 \mu$ poise for $T \leqslant 0.8 \mathrm{~K}$ ) at $1 \mathrm{GHz}$ is much larger than the pore radius $R$. We are then dealing with low frequency regime [10] in our porous Vycor glass and $f$ related to the Poiseuille flow through the porous medium. As a result of the solution of the dispersion relation deduced from the compatibility of (4) and (5), the slow mode (fluid) is overdamped and the fast one (solid) has a velocity $V$ and an attenuation $\alpha_{\text {Tot }}$ :

$$
V^{2}=\frac{E_{1}+E_{\mathrm{v}}}{\rho_{1}+\rho_{\mathrm{v}}} \quad \alpha_{\mathrm{Tot}}=\frac{\omega^{2}}{V^{3}} \frac{a_{1}+a_{\mathrm{v}}}{\rho_{1}+\rho_{\mathrm{v}}} .
$$

For liquid helium

$$
\left(\rho_{1}=x \rho=0.045 \mathrm{~g} / \mathrm{cm}^{3} ; v_{1}=c=235 \mathrm{~m} / \mathrm{s}\right)
$$

in porous Vycor glass

$$
\left(\rho_{\mathrm{v}}=1 \mathrm{~g} / \mathrm{cm}^{3} ; v_{\mathrm{v}}=3300 \mathrm{~m} / \mathrm{s}\right)
$$

expression (6) is much simpler :

$$
V=v_{\mathrm{v}} \quad \alpha_{\mathrm{Tot}}=\alpha_{\mathrm{v}}+\frac{\rho c^{3}}{\rho_{\mathrm{v}} v_{\mathrm{v}}^{3}} \alpha_{\mathrm{He}} .
$$

A last comment about the penetration length $\delta$, $\delta \gg R$ means [12] that the pure mode of helium confined in Vycor is fourth sound, but at low temperatures $(T<1 \mathrm{~K})$ the fourth sound velocity is very close to the first sound one.

4. Experimental results. - Our experimental data for helium confined in Vycor 1 are presented in figure 1. We have plotted the attenuation $\left(\alpha_{\text {Tot }}-\alpha_{v}, \alpha_{v}\right.$ from [1]) divided by the frequency $\omega / 2 \pi$ for different frequencies versus the square of the temperature $T$. The results for Vycor 2 can be plotted as well in the same curve. As an evidence

- the attenuation of sound is proportional to the frequency ;

- up to $T=0.8 \mathrm{~K}$ the temperature dependence is quadratic;

- the experimental value of the normal density we deduce through eqs. (7) and (3) is

$$
\left.\frac{\rho_{\mathrm{np}}}{\rho}\right|_{\exp }=(2 \pm 0.5) \cdot 10^{-2} T^{2}
$$

for both types of Vycor.

The $T^{2}$ dependence clearly shows that the dimensionality is effectively $1(N=1$ in eq. (1)) in the behaviour of helium confined in Vycor at low temperatures. The prefactor in eq. (1) has been calculated by Padmore [3] :

$$
\frac{\rho_{\mathrm{np}}^{[1]}}{\rho}=\frac{\left(k_{\mathrm{B}} T\right)^{2}}{3 \rho \hbar c^{3} R^{2}}
$$




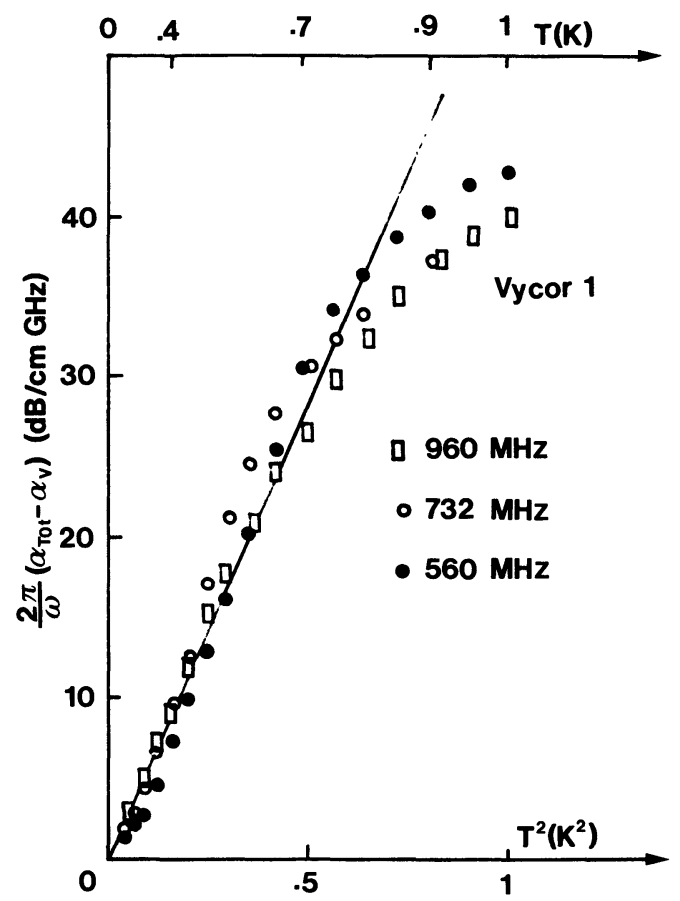

Fig. 1. - The ultrasonic attenuation $\left(\alpha_{\mathrm{T} \alpha}-\alpha_{\mathrm{v}}\right)$ divided by the frequency $\omega / 2 \pi$ is plotted versi's $T^{2}$. From the slope of the straight line through the data, we deduce the value $\rho_{\mathrm{np}}$ of the contribution of the phonons to the normal density (see text).

When applied to our samples eq. (9) gives $(8 \pm 1) \cdot 10^{-6} T^{2}$ for Vycor 1 and $(30 \pm 2) \cdot 10^{-6} T^{2}$ for Vycor 2. These numerical values are three orders of magnitude smaller than our measurement and also are different for the two samples; this last feature is not observed experimentally. Moreover our value $(2 \pm 0.5) \cdot 10^{-2} T^{2}$ is in reasonable agreement with the experimental data $\left(3 \times 10^{-2} T^{2}\right)$ of fourth sound measurements [4]. In order to reconcile theory and experiment we can try to take into account the effect of inhomogeneity of the helium due to van der Waals forces [13] : there is a solid layer $(\sim 10 \AA)$ and also some inhomogeneity in the sound velocity due to pressure. Any one of these effects is greater than some per cent and the best we can gain is a factor of two but never three orders of magnitude. Therefore a discrepancy subsists between theory and experiment, not only for the prefactor but also for the radius dependence $\left(\rho_{\mathrm{np}}\right.$ is not proportional to $\left.R^{-2}\right)$.

The last feature of our experiments concerns the attenuation of sound in Vycor partially filled with helium, i.e. with a film on it. Due to our experimental device we are not able to control the film thickness, but for certain thicknesses we do observe an enhancement of the attenuation of sound up to a factor 10 larger than in the case of Vycor full of helium. The existence of a free surface permits some surface modes, called ripplons. The contribution of these modes [5] to the normal density has the same form as in eq. (9) but with the velocity $v_{\mathrm{R}}$ of the ripplon instead of $c$. As was quoted by Saam and Coole [5] there is an instability for the film in a cylindrical pore and for thicknesses close to this instability the velocity $v_{R}$ decreases rapidly (to zero). At such a decrease in $v_{R}$ there is a corresponding enhancement of the normal density $\left(\rho_{\mathrm{n}} \propto v_{\mathrm{R}}^{-3}\right)$ which is able to interpret the enhancement we observe in the attenuation of sound for a film of helium in Vycor glass.

5. Conclusion. - We have measured the attenuation of sound in liquid helium confined in porous Vycor glass. Our data clearly show the one-dimensional behaviour of the normal density, but our numerical value of the prefactor, in accord with a previous experiment, is three orders of magnitude larger than the theoretical prediction.

\section{References}

[1] Lambert, B., Perzynski, R. and Salin, D., J. Physique Lett. 41 (1980) L-19.

[2] Landau, L., J. Phys. U.S.S.R. 5 (1941) 71.

[3] Padmore, T. C., Phys. Rev. Lett. 28 (1972) 1512.

[4] Kiewett, C., Hall, H. and RePPy, J., Phys. Rev. Lett. 35 (1976) 1286.

[5] SaAm, W. F. and Coole, M. W., Phys. Rev. B 11 (1975) 1086.

[6] Khalatnikov, I. M. and Chernikova, D. M., Sov. Phys. JETP 49 (1965) 1957; ibid. 50 (1965) 411.

[7] Maris, H. J., Phys. Rev. A 8 (1973) 2629.
[8] Matseev, Yu. A., Sov. Phys. JETP 38 (1974) 582.

[9] Salin, D., Thèse, Université Pierre-et-Marie-Curie, Paris (1979).

[10] Biot, M. A., J. Acoust. Soc. Am. 28 (1956) 168.

[11] Bacri, J.-C. and Rajaonarison, R., J. Physique Lett. 40 (1979) L-5.

[12] Adamenko, I. N. and Kaganov, M. I., Sov. Phys. JetP 26 (1968) 394.

[13] Lambert, B., Perzynski, R., Salin, D. and Joffrin, J., Low Temp. Phys. 28 (1977) 359. 